IFUP-TH 2010/46

\title{
Vacuum Cherenkov Radiation In Quantum Electrodynamics With High-Energy Lorentz Violation
}

\author{
Damiano Anselmi ${ }^{a, b}$ and Martina Taiuti ${ }^{b, c}$ \\ ${ }^{a}$ Institute of High Energy Physics, Chinese Academy of Sciences, \\ 19 (B) Yuquanlu, Shijingshanqu, Beijing 10049, China, \\ ${ }^{b}$ Dipartimento di Fisica "Enrico Fermi", Università di Pisa, \\ Largo B. Pontecorvo 3, I-56127 Pisa, Italy, \\ ${ }^{c}$ INFN, Sezione di Pisa, \\ Largo B. Pontecorvo 3, I-56127 Pisa, Italy \\ damiano.anselmi@df.unipi.it, martina.taiuti@df.unipi.it
}

\begin{abstract}
We study phenomena predicted by a renormalizable, CPT invariant extension of the Standard Model that contains higher-dimensional operators and violates Lorentz symmetry explicitly at energies greater than some scale $\Lambda_{L}$. In particular, we consider the Cherenkov radiation in vacuo. In a rather general class of dispersion relations, there exists an energy threshold above which radiation is emitted. The threshold is enhanced in composite particles by a sort of kinematic screening mechanism. We study the energy loss and compare the predictions of our model with known experimental bounds on Lorentz violating parameters and observations of ultrahigh-energy cosmic rays. We argue that the scale of Lorentz violation $\Lambda_{L}$ (with preserved CPT invariance) can be smaller than the Planck scale, actually as small as $10^{14}-10^{15} \mathrm{GeV}$. Our model also predicts the Cherenkov radiation of neutral particles.
\end{abstract}




\section{Introduction}

Lorentz symmetry is one of the most precise symmetries in nature [1. Nevertheless, the possibility that it might be violated at high energies or large distances is still open and has been widely investigated. If we assume that Lorentz symmetry is not exact, several phenomena that are otherwise forbidden can occur. Examples are the Cherenkov radiation in vacuo and the photon decay into an electron-positron pair. Studying phenomena of this type and comparing predictions with experimental data, we can look for signs of Lorentz violation and put bounds on the values of Lorentz violating parameters.

From the theoretical point of view, it is interesting to know that if Lorentz symmetry is (explicitly) violated at high energies, vertices that are non-renormalizable by power counting can become renormalizable by a modified power-counting criterion, which assigns different weights to space and time [2]. Consistent models, where the dispersion relations are modified by higher powers of momenta, can contain operators of higher dimensions, such as two-scalar-two-fermion vertices and four-fermion vertices; they are multiplied by inverse powers of some energy $\Lambda_{L}$, which can be interpreted as the scale of Lorentz violation. Lorentz violating gauge theories [3, 4] can be formulated, as well as Lorentz violating extensions of the Standard Model [5, 6], which we call, for brevity, LVSM. In the common perturbative framework, these theories are unitary, local, polynomial and causal.

Various phenomena that are forbidden in Lorentz invariant theories, but allowed in Lorentz violating ones, have been studied in the literature, mainly using the modified dispersion relations of low-energy effective models. Here we plan to study some of those phenomena in the realm of the LVSM, where the dispersion relations are crucial for renormalizability, therefore more constrained and valid, in principle, at arbitrarily high energies (when gravity is switched off). Our purpose is to derive bounds on the magnitude of $\Lambda_{L}$. We believe that the scale of Lorentz violation may be smaller than the Planck scale. If this were true, our understanding of physics around the Planck scale, in particular quantum gravity, would have to be reconsidered from scratch.

We assume that CPT is preserved (or that it is violated at energies much larger than $\Lambda_{L}$ ). The value of $\Lambda_{L}$ originally suggested in ref. [5] from considerations about neutrino masses and bounds on proton decay was $\Lambda_{L} \sim 10^{14}-10^{15} \mathrm{GeV}$. (In the appendix we briefly review those arguments and the minimal LVSM.) In this paper we show that such values are indeed compatible with experimental data on Lorentz violating phenomena.

Experimental bounds on the parameters that multiply higher-dimensional operators can be read from the tables of Kostelecky and Russell [1. At present, the best results belong to the photon sector, and concern the quadratic terms

$$
F_{k \lambda} \partial_{\alpha_{1}} \cdots \partial_{\alpha_{n}} F_{\mu \nu}
$$


In particular, from astrophysical birefringence and astrophysical dispersion it is found that the coefficients of the terms of dimensions 6 and 8 are bounded by

$$
\lesssim 10^{-29} \mathrm{GeV}^{-2} \text { and } \lesssim 10^{-25} \mathrm{GeV}^{-4}
$$

respectively. Interpreting such coefficients as $\sim 1 / \Lambda_{L}^{2}$ and $\sim 1 / \Lambda_{L}^{4}$ we see that these experimental data are consistent with our claim that $\Lambda_{L}$ could be as small as $10^{14}-10^{15} \mathrm{GeV}$.

Under some assumptions, ultrahigh-energy cosmic rays have been claimed to raise the bound on $\Lambda_{L}$ well above the Planck scale [7]. However, the nature of ultrahigh-energy cosmic rays has not been firmly established, yet, so it is not obvious how to use them to put unambiguous bounds on the scale of Lorentz violation. In this paper we give several scenarios that are consistent with a value of $\Lambda_{L}$ well below the Planck scale, assuming that ultrahigh-energy cosmic rays are protons or heavy nuclei. For our purposes, it will be sufficient to restrict to the minimal QED subsector of the LVSM, which we call LVQED.

We focus on the Cherenkov radiation in vacuo. For a very general class of dispersion relations we prove that there exists an energy threshold above which radiation is emitted and below which it is not emitted. Quite interestingly, the threshold is enhanced in composite particles by a sort of kinematic screening mechanism. We study the energy loss as a function of time and prove that in all cases of our interest it is so rapid that the radiation is practically governed by pure kinematics. Our models also predict the Cherenkov radiation of neutral particles.

The paper is organized as follows. In section 2 we present the LVQED model we are going to study and some basic formulas. In section 3 we study the Cherenkov radiation in the lowenergy expansion. From section 4 onwards we investigate situations where the standard lowenergy expansion does not apply. Some results can be derived using exact dispersion relations. For other purposes a different kind of expansion can be used. In section 4 we study kinematic constraints and derive the energy threshold for Cherenkov radiation. In section 5 we compare two typical scenarios with experimental data. In section 6 we study composite particles and show that compositeness favors larger thresholds. In section 7 we discuss the Cherenkov radiation of neutrons and neutrinos, while section 8 contains our conclusions.

\section{Preliminaries}

In this section we write the models we are going to study and derive a general formula for the energy loss per unit time.

The LVQED model we consider is the minimal QED subsector of the LVSM recalled in the 
appendix, to which we refer for the notation. Its Lagrangian reads

$$
\begin{aligned}
\mathcal{L}= & \mathcal{L}_{F}+\bar{\psi}\left(i \gamma^{0} D_{0}+\frac{i b_{0}}{\Lambda_{L}^{2}} \bar{D}^{3}+i b_{1} \bar{D}-m-\frac{b^{\prime}}{\Lambda_{L}} \bar{D}^{2}\right) \psi \\
& +\frac{e}{\Lambda_{L}} \bar{\psi}\left(b^{\prime \prime} \sigma_{i j} F_{i j}+\frac{b_{0}^{\prime}}{\Lambda_{L}} \gamma_{i} \partial_{j} F_{i j}\right) \psi+i e \frac{b_{0}^{\prime \prime}}{\Lambda_{L}^{2}} F_{i j}\left(\bar{\psi} \gamma_{i} \frac{\overleftrightarrow{D}_{j}}{2} \psi\right)
\end{aligned}
$$

where the covariant derivative is $D_{\mu}=\partial_{\mu}+i e A_{\mu}, \sigma_{\mu \nu}=-i\left[\gamma_{\mu}, \gamma_{\nu}\right] / 2$. Moreover,

$$
\mathcal{L}_{F}=\frac{1}{2} F_{0 i}^{2}-\frac{1}{4} F_{i j}\left(\tau_{2}-\tau_{1} \frac{\bar{\partial}^{2}}{\Lambda_{L}^{2}}+\tau_{0} \frac{\left(-\bar{\partial}^{2}\right)^{2}}{\Lambda_{L}^{4}}\right) F_{i j}
$$

is the Lagrangian of free photons. The quantization of this theory has been studied in ref. [8]. More details can be found there, together with an analysis of its renormalization. The dispersion relations of fermions and photons are

$$
E\left(\bar{p}^{2}\right)=\sqrt{\bar{p}^{2}\left(b_{1}+\frac{b_{0}}{\Lambda_{L}^{2}} \bar{p}^{2}\right)^{2}+\left(\frac{b^{\prime}}{\Lambda_{L}} \bar{p}^{2}+m\right)^{2}}, \quad \omega\left(\bar{k}^{2}\right)=\sqrt{\tau_{2} \bar{k}^{2}+\tau_{1} \frac{\left(\bar{k}^{2}\right)^{2}}{\Lambda_{L}^{2}}+\tau_{0} \frac{\left(\bar{k}^{2}\right)^{3}}{\Lambda_{L}^{4}}},
$$

respectively.

The low-energy limit of (2.1) is

$$
\mathcal{L}_{\text {low }}=\frac{1}{2} F_{0 i}^{2}-\frac{\tau_{2}}{4} F_{i j}^{2}+\bar{\psi}\left(i \gamma^{0} D_{0}+i b_{1} \bar{D}-m\right) \psi,
$$

which formally coincides with the Lagrangian of QED in a medium. The parameters $\tau_{2}$ and $b_{1}$ are related to the dielectric constant $\varepsilon$ and the magnetic permeability $\mu$ by the formulas

$$
\tau_{2}=\frac{\varepsilon}{\mu}, \quad b_{1}=\varepsilon
$$

Moreover,

$$
n=\sqrt{\varepsilon \mu}=\frac{b_{1}}{\sqrt{\tau_{2}}}
$$

is the refractive index. Performing the replacements (2.4) and the rescalings

$$
x^{i} \rightarrow \varepsilon x^{i}, \quad A_{i} \rightarrow \frac{A_{i}}{\varepsilon}, \quad \psi \rightarrow \frac{\psi}{\varepsilon^{3 / 2}},
$$

in the action of (2.3), we obtain the more common Lagrangian

$$
\mathcal{L}_{\text {medium }}=\frac{\varepsilon}{2} F_{0 i}^{2}-\frac{1}{4 \mu} F_{i j}^{2}+\bar{\psi}(i \not D-m) \psi
$$

We use for (2.5) the gauge-fixing term of Lorenz type

$$
\mathcal{L}_{\mathrm{GF}}=-\frac{1}{2 \mu}\left(\varepsilon \mu \partial_{0} A_{0}-\partial_{i} A_{i}\right)^{2}
$$


We assume that a particle above threshold continuously loses its energy through the process $e \rightarrow e \gamma$. Then the emitted radiation is made of a large number of low-frequency photons. The particle remains above threshold, but its energy asymptotically tends to the threshold value. This conservative assumption is sufficient for our purposes. Indeed, we are going to show that in all cases we are interested in the energy loss calculated in this way is so rapid that we can assume that the particle (practically) reaches the threshold instantaneously. Other types of emission occur. For example, the model (2.1) also contains vertices with two fermions and two or more photons, which allow elementary processes such as $e \rightarrow e \gamma \gamma$ and $e \rightarrow e \gamma \gamma \gamma$. It may also happen [9] that the particle loses most of its energy emitting a single sufficiently energetic photon, or a finite number of photons. Then the deceleration is not continuous. These effects can increase the rate of energy loss, but do not affect the conclusions of this paper.

It is convenient to derive a general formula for the energy loss per unit time without making assumptions on the dispersion relations. It will be applied to both (2.1) and (2.3). Consider a charged fermion of energy $E$ and momentum $p$ emitting a photon of frequency $\omega$. Call $E^{\prime}$ and $p^{\prime}$ the energy and momentum of the fermion after emission. The expression of the differential width is

$$
\mathrm{d} \Gamma=\frac{1}{2 E} \overline{|\mathcal{M}|^{2}}(2 \pi) \delta\left(E-\omega-E^{\prime}\right)(2 \pi)^{3} \delta^{3}\left(\mathbf{p}-\mathbf{k}-\mathbf{p}^{\prime}\right) \frac{\mathrm{d}^{3} \mathbf{k}}{2 \omega(2 \pi)^{3}} \frac{\mathrm{d}^{3} \mathbf{p}^{\prime}}{2 E^{\prime}(2 \pi)^{3}},
$$

where $\overline{|\mathcal{M}|^{2}}$ is the squared modulus of the transition amplitude, summed over the final states and averaged over the initial states.

As usual, the integral over $\mathbf{p}^{\prime}$ is done eliminating the delta function associated with momentum conservation. The surviving integral is reduced to an integral over $\omega$ and $u=\cos \theta, \theta$ being the angle between the momentum of the incoming fermion and the momentum of the emitted photon. Next, the delta function of energy conservation can be used to perform the $u$-integral. It gives $u$ as a function of $p$ and $k$. Finally, the condition $|u(p, k)| \leqslant 1$ determines the range of the final $k$-integration. We find

$$
\frac{\mathrm{d} \Gamma}{\mathrm{d} \omega}=\frac{\overline{|\mathcal{M}|^{2}}}{16 \pi E p} \frac{k}{\omega} \frac{\mathrm{d} k}{\mathrm{~d} \omega} \sum_{u^{*}} \frac{1}{\left|\frac{E^{\prime}}{p^{\prime}} \frac{\mathrm{d} E^{\prime}}{\mathrm{d} p^{\prime}}\right|_{u=u^{*}}},
$$

where the sum is over the solutions $u^{*}(p, k)$ to the condition of energy conservation. In the case of (2.5), the solution is unique. Instead, the dispersion relations of our Lorentz violating models admit multiple solutions, in general. Yet, the solution remains unique under quite reasonable assumptions (see section 4 ). In this case the $k$-range is of the standard form $0 \leqslant k \leqslant k_{\max }$, for some $k_{\max }$.

The differential width can be used to calculate the energy loss per unit time, using the formula

$$
\frac{\mathrm{d} E}{\mathrm{~d} t}=-\int_{0}^{\omega_{\max }} \omega \frac{\mathrm{d} \Gamma}{\mathrm{d} \omega} \mathrm{d} \omega
$$

where $\omega_{\max }=\omega\left(k_{\max }^{2}\right)$. 


\section{Cherenkov radiation in QED}

In this section we study the energy loss of charged particles in empty space due to the violation of Lorentz symmetry, first in the low-energy theory (2.3) and later in the low energy expansion of the complete theory (2.1). We then apply our formulas to ultrahigh-energy cosmic rays. The Cherenkov radiation in vacuo has been studied by various authors [9, 10, 11, 12, Some results of this section are already available in the literature, others are new.

The Cherenkov radiation occurs if $n>1$, which we assume here. (If $n<1$ a sufficiently energetic photon can decay into an electron-positron pair, see for example [10]. However, we are not going to study that phenomenon in this paper). We use the notation (2.5) and work out exact formulas without assuming that $n$ is close to 1 , so our results can be also applied to the Cherenkov radiation of charged particles in true media.

The propagators derived from (2.5) and (2.6) are

$$
\left\langle A_{\mu}(k) A_{\nu}(-k)\right\rangle=\frac{i}{\varepsilon} \frac{\operatorname{diag}\left(-1 / n^{2}, \mathbb{1}\right)}{\omega^{2}-\left(\mathbf{k}^{2} / n^{2}\right)+i 0}, \quad\langle\psi(p) \bar{\psi}(-p)\rangle=i \frac{\not p+m}{p^{2}-m^{2}+i 0},
$$

where $k=(\omega, \mathbf{k})$. From these expressions we can read the formulas for the sums over polarization states:

$$
\sum_{\lambda} \varepsilon_{\mu}^{(\lambda)} \varepsilon_{\nu}^{(\lambda) *}=\frac{1}{\varepsilon} \operatorname{diag}\left(-1 / n^{2}, \mathbb{1}\right), \quad \sum_{s} u_{s}(p) \bar{u}_{s}(p)=\not p+m, \quad \sum_{s} v_{s}(p) \bar{v}_{s}(p)=\not p-m .
$$

After a small amount of work we find that formula (2.8) gives

$$
\frac{\mathrm{d} \Gamma}{\mathrm{d} \omega}=\frac{\mu \alpha}{2 E p}\left\{\frac{n^{2}-1}{n^{2}}\left[2 E(E-\omega)+\frac{\omega^{2}}{2}\left(n^{2}+1\right)\right]-2 m^{2}\right\},
$$

with

$$
\omega \leqslant \omega_{\max }=\frac{2(n p-E)}{n^{2}-1}, \quad \frac{1}{n} \leqslant v \equiv \frac{p}{E}<1 .
$$

In the limit $v \ll 1, \omega \ll E$, formula (3.2) agrees with the classic one, see e.g. [13]. The energy loss (2.9) per unit time is

$$
\frac{\mathrm{d} E}{\mathrm{~d} t}=-\frac{\alpha m^{2} \mu(n v-1)^{3} P(v)}{3 n^{2}\left(n^{2}-1\right)^{3} v\left(1-v^{2}\right)},
$$

where

$$
P(x)=3 n\left(3 n^{2}-1\right) x-\left(5 n^{2}+1\right) .
$$

The result (3.3) agrees with the one found by Klinkhamer and Schreck in ref. [12]. We can rewrite it as a differential equation for the velocity as a function of time:

$$
\frac{\mathrm{d} v}{\mathrm{~d} t}=-\frac{\alpha m \mu(n v-1)^{3} \sqrt{1-v^{2}} P(v)}{3 n^{2}\left(n^{2}-1\right)^{3} v^{2}} .
$$




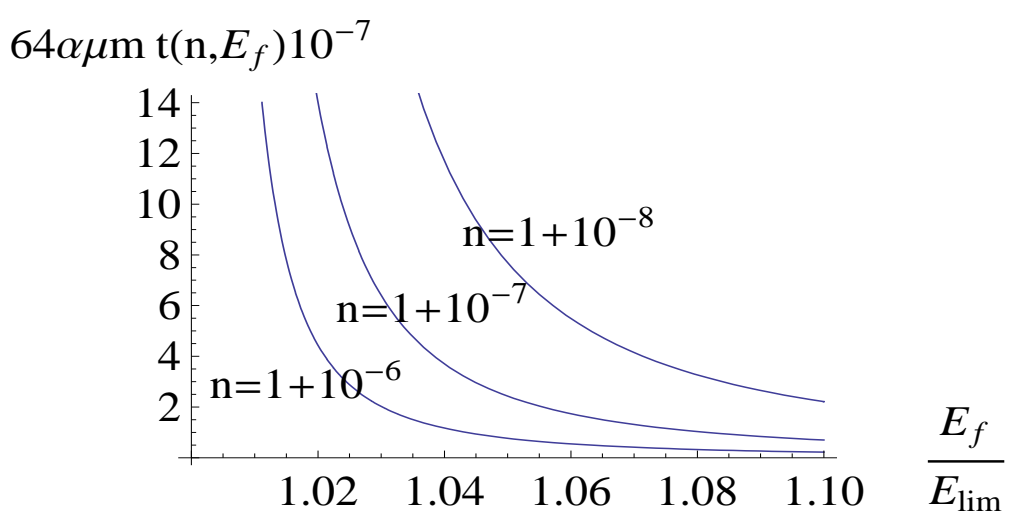

Figure 1: Radiation time

The energy decreases to the asymptotic limit

$$
E_{\lim }=\frac{m n}{\sqrt{n^{2}-1}}
$$

which corresponds to the asymptotic velocity $v_{\lim }=1 / n$. Equation (3.4) can be integrated around $v=1$, but not around $v_{\text {lim }}$. This means that a particle with infinite energy radiates to some final energy $E_{f}=m / \sqrt{1-v_{f}^{2}}$ in a finite amount of time $t\left(n, E_{f}\right)$, but reaches the energy limit (3.5) only after an infinite amount of time: $t\left(n, E_{\lim }\right)=\infty$.

Radiation time Solving (3.4) we find

$$
\begin{aligned}
t\left(n, E_{f}\right)= & \frac{3 n\left(n^{2}-1\right)\left(3-n v_{f}\right)}{16 \alpha E_{f} \mu\left(n v_{f}-1\right)^{2}}+\frac{3\left(25 n^{4}+14 n^{2}-3\right)}{64 \alpha m \mu \sqrt{n^{2}-1}} \ln \frac{n-v_{f}+\sqrt{\left(n^{2}-1\right)\left(1-v_{f}^{2}\right)}}{n v_{f}-1} \\
& -\frac{9\left(3 n^{2}-1\right)\left(5 n^{2}+1\right)^{2}}{64 \alpha m \mu \sqrt{n^{2}-1} \sqrt{P_{+} P_{-}}} \ln \frac{v_{f} P\left(1 / v_{f}\right)+\sqrt{P_{+} P_{-}\left(n^{2}-1\right)\left(1-v_{f}^{2}\right)}}{P\left(v_{f}\right)},
\end{aligned}
$$

where

$$
P_{ \pm}=9 n^{2} \pm 4 n+1
$$

Plotting (3.6) for various values of $n$ close to 1 , we can see that the energy decrease has a regular shape (see Fig. 1). For all our practical purposes the particle loses "all" its energy during some finite effective radiation time. However, since the decay is not exponential, the radiation time must be defined in an unconventional way.

Assume that the maximum observed energy of a certain class of particles is $E_{\text {obs }} \gg m$. Then, if we knew that $E_{\mathrm{obs}} \leqslant E_{\text {lim }}$ we would obtain the bound

$$
n \leqslant \frac{1}{\sqrt{1-\frac{m^{2}}{E_{\mathrm{obs}}^{2}}} .}
$$


Since we cannot exclude that our $E_{\text {obs }}$ is greater than $E_{\text {lim }}$, we must content ourselves with a worse bound. However, we can show that the decay is so fast that the "worse" bound is for all practical purposes as good as (3.7).

We consider ultrahigh-energy cosmic rays, for which we take the highest observed energy $E_{\mathrm{obs}}=3 \cdot 10^{11} \mathrm{GeV}$ [14]. In most part of this paper, we assume that they are protons or iron atoms moving in empty space. As far as the fine-structure constant $\alpha$ is concerned, we use the value $1 / 116$, namely the Standard-Model value of the running coupling at $E_{\text {obs }}$, calculated using the beta functions of ref. [15], the value of $\alpha\left(M_{z}\right)$ of [16] and the values of $M_{Z}$ and $\sin \theta_{W}\left(M_{Z}\right)$ from Particle Data Group [17]. We neglect the running of $\alpha$ from $E_{\text {obs }}$ to $\Lambda_{L}$, because it does not affect out estimates very much. Indeed, $\alpha \sim 1 / 113$ at $10^{14} \mathrm{GeV}$, and $\alpha \sim 1 / 106$ at $10^{19} \mathrm{GeV}$.

Writing $E_{\text {obs }}=r E_{\text {lim }}$, with $r>1$, we have

$$
n(r)=\frac{1}{\sqrt{1-\frac{m^{2} r^{2}}{E_{\mathrm{obs}}^{2}}} .}
$$

The age of ultrahigh-energy cosmic rays cannot exceed

$$
t_{f}(r)=t\left(n(r), E_{\mathrm{obs}}\right)
$$

since when they were created they certainly had a finite energy. Plotting $t_{f}(r)$, we see that it is a decreasing function of $r$ and tends to infinity for $r \rightarrow 1^{+}$. If ultrahigh-energy primaries are protons, it is easy to check that for $r^{2}=2$ and $\mu=1$, for example, the time $t_{f}$ is just $\sim 10^{-10}$ seconds, which means that the particle loses all its energy down to $E_{\text {obs }}$ in a few centimeters. Since it certainly covers larger distances, we must have $r<\sqrt{2}$, therefore

$$
n \sim 1+\frac{r^{2}}{2} 10^{-23}<1+10^{-23} .
$$

Lowering $r^{2}$ does not improve this bound so much, so we do not need to struggle to make $r$ as close as we can to 1 and $t_{f}(r)$ equal to the age of the Universe (or the time of some intergalactic travel).

If ultrahigh-energy primaries are iron atoms we obtain the weaker bound

$$
n<1+3 \cdot 10^{-20}
$$

and $t_{f} \sim 4 \cdot 10^{-14}$ sec.

In summary, for our purposes the energy loss is so rapid that we do not make any relevant mistake if we use (3.7).

$1 / \Lambda_{L}$-corrections Our model (2.1) predicts corrections to the results found above, which can be calculated expanding in powers of $m / \Lambda_{L}$. To illustrate integrability properties we consider 
$\mathrm{d} t / \mathrm{d} v$, instead of $\mathrm{d} v / \mathrm{d} t$. The first correction to $\mathrm{d} t / \mathrm{d} v$ is

$$
\Delta \frac{\mathrm{d} t}{\mathrm{~d} v}=\frac{3 \mu v^{2}\left(n^{2}-1\right)^{2}\left(48 b^{\prime \prime} n^{4}(n v-1)^{2}+b^{\prime} P_{2}(v)\right)}{\alpha \Lambda_{L} n^{2}(n v-1)^{4} P(v)^{2} \sqrt{1-v^{2}}},
$$

where

$$
P_{2}(x)=-3 n^{2}\left(3 n^{4}+8 n^{2}-3\right) x^{2}+2 n\left(23 n^{4}+1\right) x-25 n^{4}+1
$$

and $v$ still stands for the uncorrected expression

$$
v=\sqrt{1-\frac{m^{2}}{E^{2}}}
$$

We see that $\Delta(\mathrm{d} t / \mathrm{d} v)$ can be integrated analytically from $v=1$ to any $v_{f}$ greater than $v_{\lim }$. We do not report the lengthy result here. On the other hand, higher corrections to $\mathrm{d} t / \mathrm{d} v$ cannot be integrated around $v=1$, because they contain factors $\left(1-v^{2}\right)^{k}$ with $k>1$ in the denominator.

The effects of $1 / \Lambda_{L^{-}}$corrections compete with those of $n-1$, so the expansion in powers of $1 / \Lambda_{L}$ is meaningful only if $n$ is not too close to one. In this section we have assumed that the powers of $n-1$ are dominant. We have seen that the energy loss is so rapid that the phenomenon is governed by pure kinematics, so corrections such as (3.8) are unnecessary. When $n$ is equal to 1 , or sufficiently close to 1 , there is no radiation to the zeroth order, or almost none, and we cannot make a standard low-energy expansion. In the next sections we study the case when the $1 / \Lambda_{L}$-effects are dominant.

\section{Effects of higher space derivatives}

The LVSM, of which (2.1) is a subsector, contains terms of higher dimensions. Under certain conditions those terms are responsible for Cherenkov radiation in vacuo even if $n$ is exactly one or smaller than one. Some of them can even cause the radiation of neutral particles. In this section we begin to study those effects. We first discuss the definition of $\Lambda_{L}$ and present our work hypothesis. Then we study the kinematics of the Cherenkov process.

Definition of $\Lambda_{L} \quad$ Each term of higher dimension contained in the LVSM can be used to define a scale of Lorentz violation. Normalizing dimensionless coefficients to one, we can write a term of this type as

$$
\frac{1}{\Lambda_{i L}^{d_{i}-4}} \mathcal{O}^{i}
$$

where $\mathcal{O}^{i}$ is a local operator of dimension $d_{i}>4$ constructed with the fields and their derivatives and $\Lambda_{i L}$ is an energy scale, which can be regarded as the scale of Lorentz violation associated with $\mathcal{O}^{i}$. 
As far as we know, the values of such $\Lambda_{i L}$ 's may significantly differ from one another. So the question is: which is the scale of Lorentz violation $\Lambda_{L}$ ? The natural answer is: the smallest $\Lambda_{i L}$, namely the smallest energy scale at which the Lorentz violation may manifest itself. Since the LVSM contains a finite number of parameters, this definition is meaningful in our approach. Yet, it is a purely theoretical definition, because no sign of Lorentz violation has been observed so far.

At the theoretical level, not all parameters of the LVSM are on the same footing: most of them could be set to zero without affecting the consistency of the model. Some parameters, on the other hand, must necessarily be nonzero, because they are crucial for renormalizability. They are the coefficients that multiply the quadratic terms of the largest dimensions of each particle: the $\tau_{0}$ 's of gauge groups and the $b_{0}$ 's of fermions. In the model (2.1) the crucial terms are

$$
-\frac{\tau_{0}}{4 \Lambda_{L}^{4}} F_{i j}\left(-\bar{\partial}^{2}\right)^{2} F_{i j}, \quad \frac{i b_{0}}{\Lambda_{L}^{2}} \bar{\psi} \bar{D}^{3} \psi
$$

while parameters such as $\tau_{1}, \tau_{2}-1, b_{1}-1$, etc. are not crucial.

We would like to set the noncrucial parameters to zero, to better isolate the effects of the crucial ones. However, we have to check whether this is consistent with renormalization.

We can distinguish parameters according to the dimensions of the operators they multiply, specifically their level $d_{i}-4$. Renormalization mixing can equalize the orders of magnitude of parameters belonging to the same level. It can also have important effects on parameters of higher levels, but not so much on those of lower levels. Indeed, the beta functions of parameters belonging to lower levels receive contributions that are suppressed by powers of $m / \Lambda_{L}$. For definiteness, consider the subset of couplings $\tau_{0,1}, b_{0}$. Observe that $\tau_{0}$ is the only parameter of level 4 , while $b_{0}$ and $\tau_{1}$ are of level 2. The beta functions have structures

$$
\beta_{\tau_{0}} \sim \alpha \tau_{0}+b_{0}^{2}+b_{0} \tau_{1}+\tau_{1}^{2}, \quad \beta_{b_{0}} \sim \alpha \tau_{1}+\alpha b_{0}+\frac{m^{2}}{\Lambda_{L}^{2}} \tau_{0}, \quad \beta_{\tau_{1}} \sim \alpha \tau_{1}+\alpha b_{0}+\frac{m^{2}}{\Lambda_{L}^{2}} \tau_{0},
$$

where we have written only the first contributing terms of each type. Since $m / \Lambda_{L}$ is around $10^{-12}$, at worst, parameters of lower levels can be consistently set to have much smaller values than parameters of higher levels. In our case, $\tau_{0}$ will be of order 1 and $b_{0}$ will be of order one or much smaller than one.

This analysis is sufficient to justify the first scenario studied in the next section. Sometimes, however, it is interesting to study cases where particular relations among parameters of the same level hold, but then the effects of renormalization on those relations need to be studied carefully. The second scenario studied in the next section provides an example of this.

To summarize, the parameters of the Lorentz violating extended Standard Model can be arranged according to a hierarchy of conceptual importance, which may or may not correspond to a hierarchy of magnitude. We take it as a work hypothesis to organize our analysis. We assume 
that the absolute values of the non-crucial parameters are as small as possible, and concentrate on the crucial ones.

The values of the crucial parameters themselves can significantly differ from one another. The largest of them defines $\Lambda_{L}$. For example, if the scale of Lorentz violation $\Lambda_{L}$ is defined by the crucial term belonging to the photon sector, namely

$$
-\frac{1}{4} F_{i j} \frac{\left(-\bar{\partial}^{2}\right)^{2}}{\Lambda_{L}^{4}} F_{i j},
$$

then we can set $\tau_{0}=1$ for the photon, and assume that all other $\tau_{0}$ 's, and the $b_{0}$ 's, are not greater than 1. This choice sounds reasonable, indeed, because the photon sector contains the best measured parameters among those multiplying operators of higher dimensions [1]. Under these assumptions, our plan is to study how small the parameters $b_{0}$ 's have to be to explain data, in particular ultrahigh-energy cosmic rays.

In the rest of this section we study the kinematics of a large class of dispersion relations. In particular, we study the threshold for Cherenkov radiation and the range of frequencies of the emitted photon.

General kinematics As before, $p$ denotes the momentum of the incoming fermion, $k$ is the momentum of the emitted photon, $u=\cos \theta$ and $\theta$ is the angle between the trajectory of the incoming fermion and the photon.

We just assume that at $p, k \neq 0$ the dispersion relations $E(p)$ and $\omega(k)$ are non-negative, have positive first derivatives (namely velocities are always positive) and non-negative second derivatives, and that at least one dispersion relation is convex:

$$
E \geqslant 0, \quad \omega \geqslant 0, \quad \frac{\mathrm{d} E}{\mathrm{~d} p}>0, \quad \frac{\mathrm{d} \omega}{\mathrm{d} k}>0, \quad \frac{\mathrm{d}^{2} E}{\mathrm{~d} p^{2}}>0, \quad \frac{\mathrm{d}^{2} \omega}{\mathrm{d} k^{2}} \geqslant 0 .
$$

These properties are obeyed by the usual relativistic and non-relativistic dispersion relations. In relativistic dispersion relations convexity holds any time the mass is non-vanishing.

Energy and momentum conservations imply

$$
E(p)=\omega(k)+E\left(p^{\prime}\right), \quad p^{\prime}=\sqrt{p^{2}+k^{2}-2 p k u} .
$$

The condition (4.4) is involved, but some inequalities that are useful for the calculation can be derived straightforwardly. For example, we have

$$
k<2 p
$$

This information is quite redundant (the precise $k$-range is determined below), but enough for the moment. It can be proved observing that $E(p)-E\left(p^{\prime}\right) \geqslant 0$ implies $p \geqslant p^{\prime}$, by the monotonicity of $E(p)$, while $k \geqslant 2 p$ would give $p^{\prime} \geqslant p$ (using $u \leqslant 1$ ). 


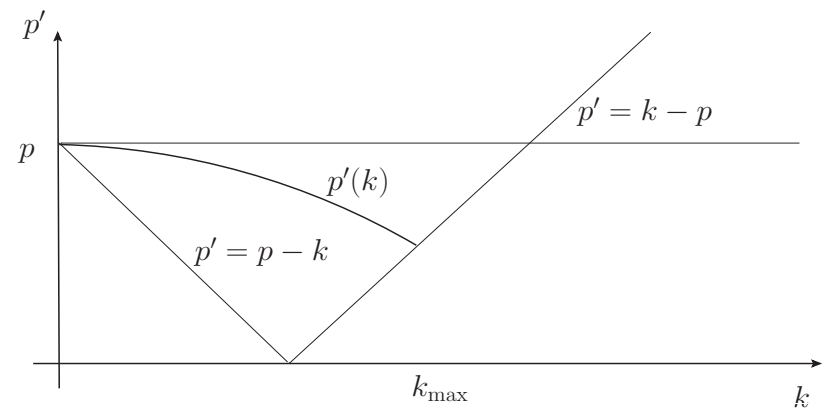

Figure 2: General kinematics

Next, consider the condition of energy conservation (4.4) in the $\left(k, p^{\prime}\right)$-plane and call its solution $p^{\prime}(k)$. For given $k$ the equation for $p^{\prime}$ reads $E\left(p^{\prime}\right)=$ constant. Since the function $E\left(p^{\prime}\right)$ is monotonic, the solution $p^{\prime}(k)$, when it exists, is unique. Second, $p^{\prime}=|\mathbf{p}-\mathbf{k}|$ and $p^{\prime} \leqslant p$ tell us that we must focus on the region

$$
|p-k| \leqslant p^{\prime} \leqslant p .
$$

Third, $k=0, p^{\prime}=p$ is a solution of (4.4), so $p^{\prime}(0)=p$.

Finally, $p^{\prime}(k)$ is monotonically decreasing and concave. These properties are proved differentiating (4.4) with respect to $k$ once and twice and using (4.3): we find, at $k \neq 0$,

$$
\frac{\mathrm{d} p^{\prime}}{\mathrm{d} k}=-\frac{\mathrm{d} \omega}{\mathrm{d} k}\left(\left.\frac{\mathrm{d} E}{\mathrm{~d} p}\right|_{p^{\prime}}\right)^{-1}<0, \quad \frac{\mathrm{d}^{2} p^{\prime}}{\mathrm{d} k^{2}}=-\left[\frac{\mathrm{d}^{2} \omega}{\mathrm{d} k^{2}}+\left.\frac{\mathrm{d}^{2} E}{\mathrm{~d} p^{2}}\right|_{p^{\prime}}\left(\frac{\mathrm{d} p^{\prime}}{\mathrm{d} k}\right)^{2}\right]\left(\left.\frac{\mathrm{d} E}{\mathrm{~d} p}\right|_{p^{\prime}}\right)^{-1}<0 .
$$

Here the notation $\left.X\right|_{p^{\prime}}$ specifies where the function $X$ has to be evaluated, namely $\left.X\right|_{p^{\prime}}=X\left(p^{\prime}\right)$.

Using these pieces of information, we can draw the picture of Fig. 2. We see that a non-trivial range of solutions exists if and only if the first derivative of $p^{\prime}(k)$ is smaller than one in modulus at $k=0$, namely

$$
-\left.\frac{\mathrm{d} p^{\prime}}{\mathrm{d} k}\right|_{0}<1 \quad \text { or, equivalently, }\left.\quad \frac{\mathrm{d} \omega}{\mathrm{d} k}\right|_{0}<\frac{\mathrm{d} E}{\mathrm{~d} p},
$$

which means that the velocity of the charged particle must be greater than a certain threshold determined by the photon dispersion relation, as in the usual case. Moreover, the $k$-range is the segment

$$
0 \leqslant k \leqslant k_{\max }(p)
$$

where $k_{\max }(p)$ is the solution of $p^{\prime}\left(k_{\max }\right)=\left|p-k_{\max }\right|$, namely it is obtained from the forward emission $u=1$.

Observe that the condition (4.6) does not depend on most parameters of $\omega(k)$. When the dispersion relations are (2.2), (4.6) does not depend on $\tau_{0}$ and $\tau_{1}$, but only $\tau_{2}$ and the parameters of the fermion dispersion relation. 


\section{$5 \quad$ Typical scenarios}

In this section we study two scenarios and their compatibility with the observation of ultrahighenergy cosmic rays and other experimental data. Our purpose is to show that there exist reasonable scenarios where the scale of Lorentz violation is smaller than the Planck scale.

From the propagators of (2.1), given in [8], we can derive the following sums over polarization states, to be used in formula (2.8):

$$
\begin{gathered}
\sum_{\lambda} \varepsilon_{\mu}^{(\lambda)} \varepsilon_{\nu}^{(\lambda) *}=\operatorname{diag}\left(-\omega^{2}\left(\bar{k}^{2}\right) / \bar{k}^{2}, \mathbb{1}\right), \quad \sum_{s} u_{s}(p) \bar{u}_{s}(p)=\not p+m+\not p\left(b_{1}-1+\frac{b_{0}}{\Lambda_{L}^{2}} \bar{p}^{2}\right)+\frac{b^{\prime}}{\Lambda_{L}} \bar{p}^{2} \\
\sum_{s} v_{s}(p) \bar{v}_{s}(p)=\not p-m+\not p\left(b_{1}-1+\frac{b_{0}}{\Lambda_{L}^{2}} \bar{p}^{2}\right)-\frac{b^{\prime}}{\Lambda_{L}} \bar{p}^{2}
\end{gathered}
$$

\subsection{First scenario}

In the first example we set all non-crucial parameters to zero apart from the mass, namely we assume

$$
\tau_{2}=1, \quad \tau_{1}=0, \quad b_{1}=1, \quad b^{\prime}=0, \quad b_{0}>0 .
$$

The results do not depend very much on the value of $\tau_{1}$ (see comment on this at the end of this section). We have the dispersion relations

$$
E\left(p^{2}\right)=\sqrt{m^{2}+p^{2}\left(1+\frac{b_{0} p^{2}}{\Lambda_{L}^{2}}\right)^{2}}, \quad \omega\left(k^{2}\right)=\sqrt{k^{2}+\tau_{0} \frac{\left(k^{2}\right)^{3}}{\Lambda_{L}^{4}}} .
$$

The inequality $b_{0}>0$ is assumed to ensure monotonicity. The condition (4.6) gives

$$
\xi^{2} \equiv \frac{m^{2} \Lambda_{L}^{2}}{6 b_{0} p^{4}}<\left(1+\frac{b_{0} p^{2}}{\Lambda_{L}^{2}}\right)^{2}\left(1+\frac{3 b_{0} p^{2}}{2 \Lambda_{L}^{2}}\right) .
$$

We are interested in the case

$$
m \ll p \ll \Lambda_{L},
$$

which can help us solve the kinematic constraints in an approximate way. Specifically, we have

$$
p \leqslant 3 \cdot 10^{11} \mathrm{GeV}, \quad \Lambda_{L} \geqslant 10^{14} \mathrm{GeV} .
$$

Within our approximation the right-hand side of (5.4) is practically 1 , so the condition for the emission of Cherenkov radiation is

$$
\xi<1
$$

which can also be expressed as an energy threshold, namely

$$
E>E_{\lim } \sim \frac{m^{1 / 2} \Lambda_{L}^{1 / 2}}{6^{1 / 4} b_{0}^{1 / 4}} .
$$


A particle above threshold radiates and loses energy till it reaches the limit value $E_{\text {lim }}$.

When $\Lambda_{L} \rightarrow \infty$ at $m$ and $p$ fixed, the condition (4.4) admits no solution, because it reduces to the kinematic relation of the Lorentz invariant theory. It is more convenient to study the limit $\Lambda_{L} \rightarrow \infty$ at $\xi$ and $p$ fixed, because in such a limit (4.4) becomes

$$
p=k+\sqrt{p^{2}+k^{2}-2 p k u},
$$

so its solution is $u=1, k \leqslant p$. For $\Lambda_{L}<\infty$ we can find an approximate solution of the form

$$
u=1-\varepsilon, \quad 0<\varepsilon \ll 1,
$$

with $k$ belonging to a certain range of values that has to be worked out. We can expand in $\varepsilon$ and $p / \Lambda_{L}$ around the $\Lambda_{L}=\infty$-solution. We find

$$
\varepsilon=\frac{b_{0}}{p k \Lambda_{L}^{2}}\left\{(p-k)\left[p^{3}-(p-k)^{3}\right]-3 \xi^{2} p^{3} k\right\} .
$$

We see that $\varepsilon$ is indeed much smaller than one, as needed for consistency. The $k$-range can be read from the condition $\varepsilon \geqslant 0$. Plotting the functions appearing in (5.8) it is easy to show that for $\xi<1$ a range of the form (4.7) exists and has $k_{\max }<p$.

The energy losses (2.9) can be worked out starting from the differential width (2.8). For the analysis of ultrahigh-energy cosmic rays it is sufficient to consider the situations $\xi^{2} \ll 1$ and $1-\xi^{2} \ll 1$. For $\xi^{2} \ll 1$ we obtain the range

$$
0 \leqslant k \leqslant p\left(1-3 \xi^{2}\right)
$$

and the energy loss

$$
\left.\frac{\mathrm{d} E}{\mathrm{~d} t}\right|_{\xi^{2} \ll 1}=-\frac{11 \alpha p^{4} b_{0}}{12 \Lambda_{L}^{2}} .
$$

For $1-\xi^{2} \ll 1$ we obtain the range

$$
0 \leqslant k \leqslant \frac{p}{2}\left(1-\xi^{2}\right),
$$

and the energy loss

$$
\left.\frac{\mathrm{d} E}{\mathrm{~d} t}\right|_{1-\xi^{2} \ll 1}=-\frac{\alpha p^{4}\left(1-\xi^{2}\right)^{3}}{4 \Lambda_{L}^{2}} b_{0} .
$$

The exact formulas depend also on $b^{\prime \prime}$. However, we have set $b^{\prime \prime}=0$, since $b^{\prime \prime}$ is not in the list of crucial parameters.

Recall that, since we have used the approximation (5.5), we cannot use (5.9) and (5.10) above $E=\Lambda_{L}$. As in the case of QED in a medium, the radiating particle takes an infinite amount of time to reach the energy limit. For our purposes it is sufficient to calculate the time the particle 
takes to radiate from energy $\Lambda_{L}$ to, say, $1.3-1.1 E_{\text {lim. }}$. It is not meaningful to approach the energy limit further, since the energies we are considering are not measured so precisely.

Now we apply our results to ultrahigh-energy cosmic rays. If $\Lambda_{L}=10^{14} \mathrm{GeV}$, protons of $3 \cdot 10^{11} \mathrm{GeV}$ emit Cherenkov radiation if $b_{0}>1.8 \cdot 10^{-19}$. If $\Lambda_{L}=10^{14} \mathrm{GeV}$ and $b_{0}=1.8 \cdot 10^{-19}$ we can use (5.9) as long as $\xi$ is small, for example down to $2 E_{\lim }\left(\xi^{2}=1 / 16\right)$. The time spent to radiate from $\Lambda_{L}$ to $2 E_{\text {lim }}$ is

$$
t_{f}^{\prime} \sim 7 \cdot 10^{-12} \text { sec. }
$$

When the energy approaches $E_{\text {lim }}$ we have to use (5.10). The particle radiates from energy $2 E_{\text {lim }}$ to $1.1 E_{\lim }$ in about

$$
t_{f}^{\prime \prime} \sim 8 \cdot 10^{-10} \mathrm{sec}
$$

The radiation time $t_{f}=t_{f}^{\prime}+t_{f}^{\prime \prime}$ is too short to be compatible with the observation of ultrahighenergy cosmic rays. Therefore, as in section 2, we may assume that the energy loss down to $E_{\text {lim }}$ occurs instantaneously any time it is allowed by kinematics.

Larger values of $b_{0}$ give smaller $t_{f}$ 's. For example, if $b_{0} \sim 1$ and $\Lambda_{L}=10^{14} \mathrm{GeV}$ particles radiate down to $E_{\text {lim }}=6 \cdot 10^{6} \mathrm{GeV}$ in a much shorter time. Integrating (5.9) from $\Lambda_{L}$ to $E_{\text {obs }}=3 \cdot 10^{11} \mathrm{GeV}$, we obtain

$$
t_{f}^{\prime} \sim 10^{-29} \mathrm{sec},
$$

while continuing down to $1.1 E_{\text {lim }}$ we have to use both (5.9) and (5.10), and get

$$
t_{f}^{\prime \prime} \sim 2 \cdot 10^{-14} \mathrm{sec}
$$

Thus, only the values $b_{0} \leqslant 1.8 \cdot 10^{-19}$ are consistent with data if $\Lambda_{L}=10^{14} \mathrm{GeV}$ and the cosmic rays are protons.

The limiting value on $b_{0}$ can be raised increasing $\Lambda_{L}$. For various values of $\Lambda_{L}$ the bounds on $b_{0}$ are

$$
b_{0 \lim }=1.8 \cdot 10^{-19+2 k} \quad \text { for } \Lambda_{L}=10^{14+k} \mathrm{GeV}
$$

and $b_{0 \text { lim }}=1$ for $\Lambda_{L}=2.4 \cdot 10^{23} \mathrm{GeV}$. When $\Lambda_{L}$ is varied between $10^{14} \mathrm{GeV}$ and the Planck scale $t_{f}$ does not change very much.

If the ultrahigh-energy cosmic rays are iron atoms we get the bounds

$$
b_{0 \lim }=5.6 \cdot 10^{-16+2 k} \quad \text { for } \Lambda_{L}=10^{14+k} \mathrm{GeV}
$$

and $b_{0 \mathrm{lim}}=1$ for $\Lambda_{L}=4.2 \cdot 10^{21} \mathrm{GeV}$.

We have also considered a variant of (5.2), with $\tau_{1}=2 \sqrt{\tau_{0}}$ instead of $\tau_{1}=0$. The radiation times are still too short and the threshold condition is exactly the same, therefore the bounds (5.11) and (5.12) are unchanged.

Most bounds we have found are very small. However, the situation improves if we take compositeness into account. Before that, we study a second scenario. 


\subsection{Second scenario}

The procedure just used is quite general, and can be used to examine other cases. We illustrate a second scenario taking the dispersion relations

$$
E\left(p^{2}\right)=\sqrt{m^{2}+p^{2}+b_{0}^{2} \frac{\left(p^{2}\right)^{3}}{\Lambda_{L}^{4}}}, \quad \omega\left(k^{2}\right)=\sqrt{k^{2}+\tau_{0} \frac{\left(k^{2}\right)^{3}}{\Lambda_{L}^{4}}},
$$

for the fermion energy and photon frequency. Here we assume that the parameters of the Lagrangian (2.1) satisfy

$$
b_{0}=-\frac{b^{\prime 2}}{2 b_{1}}, \quad b_{1}=\sqrt{1-2 \frac{m b^{\prime}}{\Lambda_{L}}}, \quad \tau_{2}=1, \quad \tau_{1}=0
$$

namely they are such that only the highest powers of momentum, which are the crucial ones for renormalization, correct the relativistic dispersion relations.

Here it is convenient to define

$$
\xi \equiv \frac{\Lambda_{L}^{2} m}{\sqrt{5}\left|b_{0}\right| p^{3}} .
$$

The approximate condition for emission is again $\xi<1$. The limit energy is

$$
E_{\lim }=\frac{\Lambda_{L}^{2 / 3} m^{1 / 3}}{5^{1 / 6}\left|b_{0}\right|^{1 / 3}}
$$

Setting $u=1-\varepsilon$ as before and $\zeta \equiv b_{0}^{2} / \tau_{0}$, we find

$$
\varepsilon \sim \frac{b_{0}^{2}}{2 p k \Lambda_{L}^{4}}\left\{(p-k)\left[p^{5}-(p-k)^{5}-\frac{k^{5}}{\zeta}\right]-5 \xi^{2} p^{5} k\right\} .
$$

For $\zeta \ll 1$ it is sufficient to consider the case $\zeta \ll\left(1-\xi^{2}\right)^{3}$, which gives the $k$-range

$$
0 \leqslant k \leqslant p\left(5 \zeta\left(1-\xi^{2}\right)\right)^{1 / 4}
$$

We find, to the lowest order in $1 / \Lambda_{L}$ and $\zeta$ (at fixed $\tau_{0}$ ), the energy loss

$$
\left.\frac{\mathrm{d} E}{\mathrm{~d} t}\right|_{\zeta \ll 1, \zeta \ll\left(1-\xi^{2}\right)^{3}}=-\frac{5 \alpha\left(1-\xi^{2}\right) \zeta^{3 / 2} \sqrt{\tau_{0}} p^{4}}{4 \Lambda_{L}^{2}} .
$$

As before, we have set $b^{\prime \prime}=0$. Instead $b^{\prime}$ must be kept, because formulas (5.14) relate it to $b_{0}$. Note that since $b_{1} \sim 1, b_{0}$ must be negative. Thus we have $b^{\prime 2} \sim-2 b_{0}=2 \sqrt{\zeta \tau_{0}}$.

Formula (5.18) can be integrated exactly. We obtain

$$
\left.E^{3}(t)\right|_{\zeta \ll 1, \zeta \ll\left(1-\xi^{2}\right)^{3}}=E_{\lim }^{3} \frac{\Lambda_{L}^{3} \cosh (\kappa t)+E_{\lim }^{3} \sinh (\kappa t)}{\Lambda_{L}^{3} \sinh (\kappa t)+E_{\lim }^{3} \cosh (\kappa t)},
$$


where

$$
\kappa=\frac{3 \sqrt{5}}{4} \alpha m \zeta
$$

and the initial condition is fixed setting $E(0)=\Lambda_{L}$. Formula (5.19) allows us to define a radiation time in a familiar way, since it contains only exponentials. We have

$$
\left.t_{f}\right|_{\zeta \ll 1, \zeta \ll\left(1-\xi^{2}\right)^{3}} \sim \frac{1}{\kappa},
$$

which is strictly speaking the time taken to reach the energy $\sim 1.1 E_{\text {lim }}$ (assuming $\Lambda_{L} \gg E_{\lim }$, which is true in the cases studied here).

If $\zeta=1$ we need to distinguish the cases $\xi^{2} \ll 1$ and $1-\xi^{2} \ll 1$. We find the $k$-ranges

$$
0 \leqslant k \leqslant p(1-\xi), \quad 0 \leqslant k \leqslant \frac{p}{3}\left(1-\xi^{2}\right)
$$

and the energy losses

$$
\left.\frac{\mathrm{d} E}{\mathrm{~d} t}\right|_{\zeta=1, \xi^{2} \ll 1}=-\frac{\alpha p^{4}\left|b_{0}\right|}{20 \Lambda_{L}^{2}},\left.\quad \frac{\mathrm{d} E}{\mathrm{~d} t}\right|_{\zeta=1, \xi^{2} \sim 1}=-\frac{\alpha\left(1-\xi^{2}\right)^{4}\left|b_{0}\right| p^{4}}{324 \Lambda_{L}^{2}},
$$

respectively.

We take $\tau_{0}=1$, which means that we assume that the scale of Lorentz violation $\Lambda_{L}$ is defined by the photon sector, precisely by the first term of (4.1). With $\Lambda_{L}=10^{14} \mathrm{GeV}$, protons of $3 \cdot 10^{11} \mathrm{GeV}$ emit Cherenkov radiation if $\left|b_{0}\right|>1.6 \cdot 10^{-7}$. If we take $b_{0}=-1.6 \cdot 10^{-7}$ the approximation $\zeta \ll\left(1-\xi^{2}\right)^{3}$ holds in the entire energy range from $\Lambda_{L}$ down to $1.1 E_{\text {lim. }}$. We find that the typical radiation time of the particles above threshold is

$$
t_{f} \sim 2 \cdot 10^{-9} \text { sec. }
$$

Again, larger values of $b_{0}$ give smaller $t_{f}$ 's. For example, if $b_{0} \sim 1$ and $\Lambda_{L}=10^{14} \mathrm{GeV}$ a proton of energy $E_{f}=3 \cdot 10^{11} \mathrm{GeV}$ has $\xi \sim 10^{-7}$, so the time it spends to radiate from energy $\Lambda_{L}$ to the final energy $E_{f} \gg E_{\text {lim }}$ can be calculated using the first formula of (5.21). We find

$$
\left.t_{f}\right|_{\zeta=1, \xi^{2} \ll 1} \sim \frac{40 \Lambda_{L}^{2}}{3 \alpha b^{\prime 2} E_{f}^{3}} .
$$

Numerically, taking $b_{0}=-1$ and $b^{\prime} \sim \sqrt{2}$, we have

$$
t_{f} \sim 2 \cdot 10^{-28} \text { sec }
$$

After this time, the cosmic rays keep radiating till they reach the limit energy, which is $E_{\lim } \sim$ 1.6 $10^{9} \mathrm{GeV}$. We can use the first formula of (5.21) as long as $\xi^{2}$ is small, for example down to $2 E_{\lim }\left(\xi^{2}=1 / 64\right)$. The time spent to radiate from $\Lambda_{L}$ to $2 E_{\lim }$ is

$$
t_{f}^{\prime} \sim 1.6 \cdot 10^{-22} \mathrm{sec} .
$$


When the energy approaches $E_{\text {lim }}$ we have to use the second formula of (5.21). The particle radiates from energy $2 E_{\text {lim }}$ to $1.1 E_{\text {lim }}$ during

$$
t_{f}^{\prime \prime} \sim 6 \cdot 10^{-20} \mathrm{sec}
$$

which is still very short.

Summarizing, we may assume that the energy loss is instantaneous, so only the values $\left|b_{0}\right| \leqslant$ $1.6 \cdot 10^{-7}$ are consistent with data at $\Lambda_{L}=10^{14} \mathrm{GeV}$. The limiting value on $\left|b_{0}\right|$ can be raised increasing $\Lambda_{L}$ and becomes 1 for $\Lambda_{L}=2.5 \cdot 10^{17} \mathrm{GeV}$. If $b_{0} \sim 1$ and $\Lambda_{L} \geq 2.5 \cdot 10^{17} \mathrm{GeV}$, protons of $3 \cdot 10^{11} \mathrm{GeV}$ do not emit Cherenkov radiation and can reach the earth. Protons above threshold have a radiation time $t_{f}^{\prime}+t_{f}^{\prime \prime}$ of about $6 \cdot 10^{-20}$ sec. We obtain the bounds

$$
\left|b_{0 \lim }\right|=1.6 \cdot 10^{-7+2 k}, \quad \text { for } \Lambda_{L}=10^{14+k} \mathrm{GeV},
$$

and $\left|b_{0}\right|=1$ for $\Lambda_{L}=2.5 \cdot 10^{17} \mathrm{GeV}$. If the ultrahigh-energy cosmic rays are instead iron atoms their observation can be explained, for example, with $\left|b_{0}\right| \sim 1,\left|b^{\prime}\right| \sim \sqrt{2}$ and $\Lambda_{L} \sim 3.4 \cdot 10^{16} \mathrm{GeV}$, or with $\left|b_{0}\right| \sim 9 \cdot 10^{-6},\left|b^{\prime}\right| \sim 4 \cdot 10^{-3}$ and $\Lambda_{L} \sim 10^{14} \mathrm{GeV}$.

Since we have assumed that the relations (5.14) hold, we must check the compatibility of $b^{\prime}$ and $b_{1}$ with present data. Using $b_{1} \sim 1, p \ll \Lambda_{L}$ and (5.14), (5.15) we get

$$
-\frac{\Lambda_{L}^{2} m}{\sqrt{5} p^{3}} \leqslant b_{0}<0, \quad\left|b^{\prime}\right| \leqslant\left(\frac{4}{5}\right)^{\frac{1}{4}} \frac{\Lambda_{L} m^{1 / 2}}{p^{3 / 2}}, \quad 1-\left(\frac{4}{5}\right)^{\frac{1}{4}}\left(\frac{m}{p}\right)^{\frac{3}{2}} \leqslant n \leqslant 1+\left(\frac{4}{5}\right)^{\frac{1}{4}}\left(\frac{m}{p}\right)^{\frac{3}{2}} .
$$

Here $p$ is the largest momentum at which the particle is known not to radiate and $n=b_{1} / \sqrt{\tau_{2}}$ is the refractive index of the vacuum "as seen by the proton". Observe that the bound on the refractive index is independent of $\Lambda_{L}$, so it cannot be improved changing the scale of Lorentz violation.

The three inequalities (5.26) are equivalent to one another. We search for the largest $\left|b_{0}\right|$ compatible with data. If $\left|b_{0}\right|$ is not small enough, $n$ may be too far from one, which may contradict existing bounds.

If $b^{\prime}>0$ we find $1-5 \cdot 10^{-18} \leqslant n<1$. At present no bounds contradict this range [1]. Instead, if $b^{\prime}<0$ we find $1<n \leqslant 1+5 \cdot 10^{-18}$. In this case, a bound exists in the literature, $n<1+6 \cdot 10^{-20}$ [12, 1], but it cannot be applied here, since it is derived from ultrahigh-energy cosmic rays themselves, which we are explaining with a different approach. Thus, the largest $\left|b_{0}\right|$ we can take is given by

$$
b_{0}=-\frac{\Lambda_{L}^{2} m}{\sqrt{5} p^{3}}
$$

Now we discuss the consistency of the dispersion relations (5.13) with renormalization. The first condition of (5.14) demands that the combination 


$$
\epsilon \equiv 2 b_{1} b_{0}+b^{2}
$$

vanish, to ensure that the dispersion relations do not contain terms proportional to the forth power of momentum. A typical case with $\epsilon$ different from zero is the first scenario already studied. The $b_{0}$-bounds of (5.11) and (5.12) tell us how small $\epsilon$ must be to have compatibility with data, in the cases of protons and iron atoms, respectively. For example, for protons $\epsilon \sim 4 \cdot 10^{-19}$ at $\Lambda_{L}=10^{14} \mathrm{GeV}$. Instead, the results found in the second scenario tell us $2 b_{1} b_{0} \sim b^{\prime 2} \sim 3.2 \cdot 10^{-7}$, which is 12 orders of magnitude larger!

For the reasons explained in the previous section, we may assume that the relations (5.14) are valid at the scale $\Lambda_{L}$, or anyway just at one energy scale. However, the scale we need to work with is $E_{\mathrm{obs}}=3 \cdot 10^{11} \mathrm{GeV}$. The $b_{0^{-}}$and $b^{\prime}$-runnings contain, among the others, terms proportional to

$$
\alpha b_{0} \ln \frac{\Lambda_{L}}{E_{\text {obs }}}, \quad \alpha b^{\prime} \ln \frac{\Lambda_{L}}{E_{\text {obs }}} .
$$

So, assuming that the cancellation $\epsilon=0$ occurs at $\Lambda_{L}$ it will not necessarily occur at $E_{\text {obs }}$, where instead we find

$$
\epsilon \sim \alpha b_{0} \ln \frac{\Lambda_{L}}{E_{\mathrm{obs}}} .
$$

The values of (5.25) give a too large $\epsilon$. Therefore, renormalization forces us to take $b_{0}$-values much smaller than the ones given in (5.25). Precisely, they are just a factor

$$
\frac{1}{\alpha \ln \frac{\Lambda_{L}}{E_{\mathrm{obs}}}}
$$

larger than the bounds (5.11) and (5.12). Still, the factor (5.28) improves the first scenario by about an order of magnitude.

\section{Composite particles}

In the previous section we have used the dispersion relations predicted by our models (A.1) and (2.1) for elementary particles, but we have applied them to composite particles, such as protons and iron atoms. In this section we investigate the dispersion relations of composite particles and discuss some phenomenological consequences. In particular, we show that in composite particles lower values of $b_{0}$ are favored.

A good starting point is to assume that at high energies the composite particle can be described in a purely kinematic way, namely by constituents moving with the same velocity $\mathbf{v}$. The effects of interactions among constituents will not be studied in this paper. Instead, the low-energy dispersion relation is just the relativistic one. Later we paste it together with the high-energy 
dispersion relation and obtain an approximate dispersion relation for the composite particle, valid both at low and high energies.

We begin considering constituents with dispersion relations

$$
E_{i}=\left|\mathbf{p}_{i}\right| \sqrt{1+\left(\frac{\eta_{i}^{2} \mathbf{p}_{i}^{2}}{\Lambda_{L}^{2}}\right)^{n-1}}
$$

Their velocities are

$$
\mathbf{v}_{i}=\frac{\mathrm{d} E_{i}}{\mathrm{~d} \mathbf{p}_{i}}=\frac{\mathbf{p}_{i}}{E_{i}}\left(1+n\left(\frac{\eta_{i}^{2} p_{i}^{2}}{\Lambda_{L}^{2}}\right)^{n-1}\right),
$$

where $p_{i}=\left|\mathbf{p}_{i}\right|$. Setting $\mathbf{v}_{i}=\mathbf{v}$ for every $i$ it is easy to derive the dispersion relation of the composite particle. Calling

$$
x_{i}=\left(\frac{\eta_{i}^{2} p_{i}^{2}}{\Lambda_{L}^{2}}\right)^{n-1}
$$

and squaring (6.2), we get the equations

$$
v^{2}\left(1+x_{i}\right)=\left(1+n x_{i}\right)^{2}
$$

Their solutions are

$$
x_{i}=\frac{v^{2}-2 n+v \sqrt{v^{2}+4 n(n-1)}}{2 n^{2}} \equiv x(v) .
$$

(It is easy to check that the other solution of the quadratic equation is not acceptable). Then we have

$$
p_{i}=\frac{x^{1 /(2 n-2)}}{\eta_{i}} \Lambda_{L}, \quad \mathbf{p}_{i}=\mathbf{v} \frac{E_{i}}{1+n x}, \quad E_{i}=\frac{x^{1 /(2 n-2)}}{\eta_{i}} \Lambda_{L} \sqrt{1+x},
$$

and therefore the total momentum and total energy are

$$
\mathbf{P}=\sum_{i} \mathbf{p}_{i}=\mathbf{v} \frac{E}{1+n x}, \quad E=\sum_{i} E_{i}=\frac{x^{1 /(2 n-2)}}{\eta} \Lambda_{L} \sqrt{1+x}
$$

where $\eta$ is defined by

$$
\frac{1}{\eta}=\sum_{i} \frac{1}{\eta_{i}}
$$

Moreover, since $\mathbf{p}_{i}=\mathbf{v} p_{i} / v$, we have also

$$
P=\sum_{i} p_{i}=\frac{x^{1 /(2 n-2)}}{\eta} \Lambda_{L}, \quad x=\left(\frac{\eta^{2} \mathbf{P}^{2}}{\Lambda_{L}^{2}}\right)^{n-1} .
$$

Thus, we find that $E$ and $\mathbf{P}$ are related by the collective dispersion relation

$$
E=|\mathbf{P}| \sqrt{1+\left(\frac{\eta^{2} \mathbf{P}^{2}}{\Lambda_{L}^{2}}\right)^{n-1}}
$$


which has the same form as the dispersion relations (6.1) of the constituents.

The crucial result is the composition rule (6.4), which states that "the weakest wins", namely if one constituent has a $\eta_{\bar{\imath}}$ much smaller than the $\eta_{i}$ 's of the other constituents, then the composite particle has a $\eta$ practically equal to $\eta_{\bar{\imath}}$.

Note that for $n=0, m_{i}=\Lambda_{L} / \eta_{i}$, we get the dispersion relation of relativistic theories, with the usual composition rule for the mass, namely $\sum_{i} m_{i}=\sum_{i} \Lambda_{L} / \eta_{i}=\Lambda_{L} / \eta=M$.

The result just found can be extended to more general dispersion relations of the form

$$
E_{i}=\left|\mathbf{p}_{i}\right| f\left(x_{i}\right), \quad x_{i}=\left(\frac{\eta_{i}^{2} \mathbf{p}_{i}^{2}}{\Lambda_{L}^{2}}\right)^{n-1} .
$$

Squaring the velocities

$$
\mathbf{v}_{i}=\frac{\mathrm{d} E_{i}}{\mathrm{~d} \mathbf{p}_{i}}=\frac{\mathbf{p}_{i}}{\left|\mathbf{p}_{i}\right|}\left(f+2(n-1) x_{i} f^{\prime}\right)
$$

and equating them to $\mathbf{v}$, we get the equations

$$
v^{2}=\left(f\left(x_{i}\right)+2(n-1) x_{i} f^{\prime}\left(x_{i}\right)\right)^{2} .
$$

Assume that the solution is unique, $x_{i}=x(v)$. Then, proceeding as above, we easily find that $E$ and $\mathbf{P}$ are related by the collective dispersion relation

$$
E=|\mathbf{P}| f(x), \quad x=\left(\frac{\eta^{2} \mathbf{P}^{2}}{\Lambda_{L}^{2}}\right)^{n-1},
$$

where $\eta$ is still given by (6.4). Again, the dispersion relation of the composite particle has the same form as the dispersion relations of its constituents.

Although the procedure just outlined is general, few dispersion relations can be treated so simply. More complicated relations generate polynomial equations of high degree, and the dispersion relation of the composite particle does not have the form of the dispersion relations of its constituents. To convince oneselves of this, it is sufficient to repeat the derivation adding mass terms to (6.1) and (6.5). Yet, masses are important for the Cherenkov effect, because they determine the energy threshold. To apply our results to ultrahigh-energy cosmic rays we argue as follows.

The dispersion relations (6.1) and (6.5) are good approximations at high energies, namely when the Lorentz violating corrections start to become important and the mass becomes negligible with respect to them. These are precisely the energies above threshold. Indeed, the emission of radiation is the first effect of the Lorentz violation in the phenomenon we are considering. Instead, at energies much smaller than the threshold the Lorentz violating corrections become negligible with respect to the mass, and the usual relativistic dispersion relation $E=\sqrt{M^{2}+p^{2}}$ holds, where $M$ is the mass of the composite particle. The full dispersion relation of the composite particle can be well approximated pasting the low- and high-energy dispersion relations. 
Now, consider ultrahigh-energy cosmic rays. In our model, setting all non-crucial parameters but the mass to zero as in (5.2), or relating the parameters as in (5.14), at high energies quarks have dispersion relations (6.5) with $n=2, f(x)=1+x$, or (6.1) with $n=3$, respectively. In both cases $\eta_{i}^{2}=\left|b_{0 i}\right|$. Thus, the dispersion relation of the composite particle can be approximated by the formulas

$$
E=\sqrt{M^{2}+p^{2}\left(1+\eta^{2} \frac{p^{2}}{\Lambda_{L}^{2}}\right)^{2}}, \quad E=\sqrt{M^{2}+p^{2}+\eta^{4} \frac{\left(p^{2}\right)^{3}}{\Lambda_{L}^{4}}},
$$

in the first and second scenarios, respectively, where $\eta$ is determined by equation (6.4).

Let us illustrate some basic properties of the composition rule (6.4). Consider the proton. Its dispersion relation has the same form as the dispersion relations of its constituents, with

$$
\left|b_{0 p}\right|=\eta^{2}=\left(\frac{2}{\left|b_{0 u}\right|^{1 / 2}}+\frac{1}{\left|b_{0 d}\right|^{1 / 2}}\right)^{-2},
$$

where $b_{0 u}$ and $b_{0 d}$ are the $b_{0}$-parameters of the quarks $u$ and $d$, respectively. If $\left|b_{0 d}\right| \ll\left|b_{0 u}\right|$ then $\left|b_{0 p}\right| \sim\left|b_{0 d}\right|$, while if $\left|b_{0 u}\right| \ll\left|b_{0 d}\right|$ then $\left|b_{0 p}\right| \sim\left|b_{0 u}\right| / 4$. This means that in composite particles, smaller values of $\left|b_{0}\right|$ are favored and the energy threshold for Cherenkov radiation is enhanced. In practice, compositeness creates a sort of screening for the emission of radiation and makes it easier to justify the small numbers found in the previous section.

We have no reason to assume that $\left|b_{0 u}\right|$ and $\left|b_{0 d}\right|$ are of the same orders. Let us first assume $\left|b_{0 u}\right| \gg\left|b_{0 d}\right|$ and normalize $\tau_{0}$ to one, as usual. Then, if ultrahigh-energy cosmic rays are protons we have

$$
\left|b_{0 p}\right| \sim\left|b_{0 d}\right|
$$

while if they are iron atoms we gain an extra factor 7396 :

$$
b_{0 \text { iron }}=\left(\frac{82}{\left|b_{0 u}\right|^{1 / 2}}+\frac{86}{\left|b_{0 d}\right|^{1 / 2}}\right)^{-2} \sim \frac{\left|b_{0 d}\right|}{7396} .
$$

In the first scenario described in the previous section the observation of ultrahigh-energy cosmic rays made of iron atoms can be explained with

$$
b_{0 d}=4.1 \cdot 10^{-12+2 k} \quad \text { for } \Lambda_{L}=10^{14+k} \mathrm{GeV}
$$

and $b_{0 d}=1$ for $\Lambda_{L}=4.9 \cdot 10^{19} \mathrm{GeV}$.

We see that when the composite structure gets more complex it becomes easier to generate small number from larger ones. Patterns like e.g.

$$
\begin{aligned}
& \tau_{0}=1, \quad b_{0 u} \sim 10^{-6}, \quad b_{0 d} \sim 4 \cdot 10^{-12}, \quad \Lambda_{L} \sim 10^{14} \mathrm{GeV}, \\
& \tau_{0}=1, \quad b_{0 u} \sim 10^{-3}, \quad b_{0 d} \sim 4 \cdot 10^{-6}, \quad \Lambda_{L} \sim 10^{17} \mathrm{GeV},
\end{aligned}
$$


are compatible with a scale of Lorentz violation smaller than the Planck scale. The values of $b_{0 u}$ have been chosen to lie somewhere in the middle between those of $\tau_{0}$ and those of $b_{0 d}$ for illustrative purposes.

In the second scenario we can gain an extra factor (5.28) and can explain the same $b_{0 \text { iron's }}$ with slightly larger $b_{0 d}$ 's:

$$
\begin{aligned}
& \tau_{0}=1, \quad b_{0 u} \sim 10^{-6}, \quad b_{0 d} \sim 5 \cdot 10^{-11}, \quad \Lambda_{L} \sim 10^{14} \mathrm{GeV}, \\
& \tau_{0}=1, \quad b_{0 u} \sim 10^{-3}, \quad b_{0 d} \sim 3 \cdot 10^{-5}, \quad \Lambda_{L} \sim 10^{17} \mathrm{GeV},
\end{aligned}
$$

Finally, if we assume $b_{0 u} \sim b_{0 d}$ we gain another factor 4 :

$$
\left|b_{0 p}\right| \sim\left|b_{0 n}\right| \sim \frac{\left|b_{0 u}\right|}{9}, \quad b_{0 \text { iron }} \sim \frac{\left|b_{0 u}\right|}{28224} .
$$

\section{Cherenkov radiation of neutrons and neutrinos}

We know that when Lorentz symmetry is violated, several otherwise forbidden phenomena are allowed. In this section we describe the Cherenkov radiation of neutral particles. Because of the Lorentz violation, photon emission is allowed by kinematics. Moreover, our models contain Pauli-like terms at the fundamental level, which couple neutral particles to the electromagnetic field. We take neutrons and neutrinos and consider both the Cherenkov radiation in a medium and the effects of higher-derivative terms.

The neutron Lagrangian is

$$
\begin{aligned}
\mathcal{L}_{\text {neutron }}= & \mathcal{L}_{F}+\bar{\psi}_{n}\left(i \gamma^{0} \partial_{0}+\frac{i b_{0 n}}{\Lambda_{L}^{2}} \bar{\phi}^{3}+i b_{1 n} \bar{\phi}-m_{n}-\frac{b_{n}^{\prime}}{\Lambda_{L}} \bar{\phi}^{2}\right) \psi_{n} \\
& +\frac{e}{\Lambda_{L}} \bar{\psi}_{n}\left(b_{n}^{\prime \prime} \sigma_{i j} F^{i j}+\frac{b_{0 n}^{\prime}}{\Lambda_{L}} \gamma_{i} \partial_{j} F_{i j}\right) \psi_{n}+i e \frac{b_{0 n}^{\prime \prime}}{2 \Lambda_{L}^{2}} F_{i j}\left(\bar{\psi}_{n} \gamma_{i} \overleftrightarrow{D}_{j} \psi_{n}\right)
\end{aligned}
$$

and the kinematics of the Cherenkov process is the one of section 4. The Cherenkov radiation can be studied adapting the results found for the proton. Indeed, after replacements of the form

$$
b^{\prime \prime}=\frac{\tilde{b}^{\prime \prime}}{e}, \quad b_{0}^{\prime}=\frac{\tilde{b}_{0}^{\prime}}{e}, \quad b_{0}^{\prime \prime}=\frac{\tilde{b}_{0}^{\prime \prime}}{e}
$$

the neutron Lagrangian matches the proton Lagrangian at $e=0$. As far as the Cherenkov radiation in a medium is concerned, we must evaluate formulas up to $\mathcal{O}\left(1 / \Lambda_{L}^{2}\right)$ corrections, perform the replacements (7.1), followed by the limit $e \rightarrow 0$ and the converse replacements. We find

$$
\frac{\mathrm{d} v}{\mathrm{~d} t}=-\frac{16 \alpha m_{n}^{3} \mu^{5} b_{n}^{\prime \prime 2}(n v-1)^{4}}{15 \Lambda_{L}^{2} n^{8}\left(n^{2}-1\right)^{5} v^{2} \sqrt{1-v^{2}}}\left[\left(5 n^{2}-1\right)\left(6 n^{2}+1\right)-4 n\left(n^{2}+1\right) v-5 n^{2}\left(5 n^{2}-1\right) v^{2}\right],
$$

with the velocity $v$ defined as in (3.9). 
We can also make an analysis similar to the one of section 5 . In the limit $b_{0 n}^{2} \ll \tau_{0}$, the analogue of (5.20) gives the typical radiation time

$$
\left.t_{f n}\right|_{b_{0 n}^{2} \ll \tau_{0}} \sim \frac{\tau_{0}}{6 \sqrt{5} b_{n}^{\prime 2}\left|b_{0 n}\right| \alpha m_{n}} .
$$

In both cases we see that the Cherenkov radiation of neutrons crucially depends on the parameter $b_{n}^{\prime \prime}$, besides $\tau_{0}$ and $b_{0 n}$. Thus, measurements cannot say much about the scales of Lorentz violation, which are our main interest in this paper, but can put bounds on the values of the parameters $b_{n}^{\prime \prime}$. Nevertheless, some aspects of the neutron Cherenkov radiation may deserve further study, since it is known that in some cases the Lorentz violation makes protons decay into neutrons [10. Then ultrahigh-energy cosmic rays could be regarded as a mixture of protons and neutrons and both particles would contribute to the emission of Cherenkov radiation.

If neutrinos are taken to be massive, their case is entirely analogous to the case of the neutron. Instead, if we neglect their mass (or assume that they are massless and that neutrino oscillations have a different explanation) the Lagrangian that describes interactions with the electromagnetic field is

$$
\mathcal{L}_{\text {neutrino }}=\mathcal{L}_{F}+\bar{\nu}\left(i \gamma^{0} \partial_{0}+\frac{i b_{0 \nu}}{\Lambda_{L}^{2}} \bar{\phi}^{3}+i b_{1 \nu} \bar{\phi}\right) \nu+\frac{e b_{0 \nu}^{\prime}}{\Lambda_{L}^{2}} \partial_{j} F_{i j}\left(\bar{\nu} \gamma_{i} \nu\right)+i e \frac{b_{0 \nu}^{\prime \prime}}{2 \Lambda_{L}^{2}} F_{i j}\left(\bar{\nu} \gamma_{i} \overleftrightarrow{\partial}{ }_{j} \nu\right)
$$

Here the effect is of higher order, because $b^{\prime \prime}$ is absent. Moreover, at vanishing mass the kinematics also changes. For example, in the case $b_{1 \nu}=\tau_{2}=1, \tau_{1}=0$, we find the $k$-range $0 \leqslant k \leqslant p$ and

$$
\frac{\mathrm{d} E}{\mathrm{~d} t}=-\frac{517 b_{0 \nu} \alpha\left(4 b_{0 \nu}^{\prime 2}+b_{0 \nu}^{\prime 2}\right) p^{8}}{10080 \Lambda_{L}^{6}} .
$$

\section{Conclusions}

If Lorentz symmetry is violated at high energies, a variety of phenomena that are normally forbidden can take place. The investigation of these phenomena can help us better address the search for signs of Lorentz violation and put bounds on the parameters of the violation. In this paper we have focused on the Cherenkov radiation in vacuo, and explored scenarios compatible with a scale of Lorentz violation $\Lambda_{L}$ smaller than the Planck scale. We have worked in the realm of the minimal Standard-Model extension that violates Lorentz symmetry at high energies, preserves CPT and rotational invariance, contains operators of higher dimensions (in particular, four-fermion vertices) and is renormalizable by weighted power counting. This SM extension offers a framework where the set of new parameters is large enough to describe the phenomena allowed by Lorentz violation, but sufficiently restricted to ensure a certain degree of predictivity.

We have studied kinematic constraints for a very general class of dispersion relations, and found an energy threshold below which particles do not radiate. We have computed the energy 
loss of particles above threshold and verified that it is so rapid that in all cases of interest the process is practically governed by pure kinematics. For different values of the scale $\Lambda_{L}$ we have studied how small certain parameters must be to have compatibility with known experimental bounds on the Lorentz violation. Interestingly, in composite particles "small numbers" are easier to explain, because the threshold for Cherenkov radiation is enhanced by a sort of kinematic screening mechanism.

Our analysis shows that there is still the possibility that the scale of Lorentz violation, with preserved CPT, is smaller than Planck scale. If confirmed, this prediction would force us to think about quantum gravity anew.

\section{Acknowledgments}

D.Anselmi wishes to thank Xinmin Zhang and the Institute of High Energy Physics of the Chinese Academy of Sciences, Beijing, for hospitality. D.Anselmi is supported by the Chinese Academy of Sciences visiting professorship for senior international scientists, grant No. 2010T2J01.

\section{Appendix: the simplest LVSM's}

For reference, in this appendix we briefly recall the simplest LVSM's and some of their features. The minimal scalarless CPT invariant LVSM schematically reads [6]

$$
\mathcal{L}_{\text {noH }}=\mathcal{L}_{F}+\mathcal{L}_{\text {kinf }}-\sum_{I=1}^{5} \frac{1}{\Lambda_{L}^{2}} g \bar{D} \bar{F}\left(\bar{\chi}_{I} \bar{\gamma} \chi_{I}\right)+\frac{Y_{f}}{\Lambda_{L}^{2}} \bar{\chi} \chi \bar{\chi} \chi-\frac{g}{\Lambda_{L}^{2}} \bar{F}^{3},
$$

where

$$
\begin{aligned}
\mathcal{L}_{F} & =\frac{1}{4} \sum_{G}\left(2 F_{0 i}^{G} F_{0 i}^{G}-F_{i j}^{G} \tau^{G}(\bar{\Upsilon}) F_{i j}^{G}\right), \\
\mathcal{L}_{\text {kinf }} & =\sum_{a, b=1}^{3} \sum_{I=1}^{5} \bar{\chi}_{I}^{a} i\left(\delta^{a b} \gamma^{0} D_{0}-\frac{b_{0}^{I a b}}{\Lambda_{L}^{2}} \bar{D}^{3}+b_{1}^{I a b} \bar{D}\right) \chi_{I}^{b},
\end{aligned}
$$

are the kinetic terms of gauge fields and fermions, respectively. Bars are used to denote space components. The "magnetic" components $F_{i j}$ of the field strengths are also denoted with $\bar{F}$. Moreover, $\chi_{1}^{a}=L^{a}=\left(\nu_{L}^{a}, \ell_{L}^{a}\right), \chi_{2}^{a}=Q_{L}^{a}=\left(u_{L}^{a}, d_{L}^{a}\right), \chi_{3}^{a}=\ell_{R}^{a}, \chi_{4}^{a}=u_{R}^{a}$ and $\chi_{5}^{a}=d_{R}^{a}, \nu^{a}=$ $\left(\nu_{e}, \nu_{\mu}, \nu_{\tau}\right), \ell^{a}=(e, \mu, \tau), u^{a}=(u, c, t)$ and $d^{a}=(d, s, b)$. The sum $\sum_{G}$ is over the gauge groups $S U(3)_{c}, S U(2)_{L}$ and $U(1)_{Y}$, and the last three terms of (A.1) are symbolic. Finally, $\bar{\Upsilon} \equiv-\bar{D}^{2} / \Lambda_{L}^{2}$, where $\Lambda_{L}$ is the scale of Lorentz violation, and $\tau^{G}$ are polynomials of degree 2. Gauge anomalies cancel out exactly as in the Standard Model [5]. 
The model (A.1) does not contain elementary scalar fields, but four-fermion vertices trigger a Nambu-Jona-Lasinio mechanism that gives masses to fermions and gauge fields, and generate Higgs bosons at low energies as composite fields [6, 18].

LVSM versions containing elementary scalar fields and incorporating the usual Higgs phenomenon exist as well. They can contain the two-scalar-two-fermion vertex $(L H)^{2} / \Lambda_{L}$ and fourfermion vertices $(\bar{\psi} \psi)^{2} / \Lambda_{L}^{2}$ at the fundamental level.

After symmetry breaking, the vertex $(L H)^{2} / \Lambda_{L}$ gives (Majorana) masses to the left-handed neutrinos. Since this vertex is the only dimension- 5 vertex present in the LVSM, it can be used to normalize the scale $\Lambda_{L}$. Assuming that the dimensionless couplings in front of it are of order one we find $\Lambda_{L} \sim 10^{14}-10^{15} \mathrm{GeV}$ [19]. Four-fermion vertices can describe proton decay. The existing bounds on proton decay can also be used to constrain $\Lambda_{L}$, and give $\Lambda_{L} \geqslant 10^{15} \mathrm{GeV}$.

Other prescriptions to normalize $\Lambda_{L}$ are considered in the paper.

\section{References}

[1] V.A. Kostelecký and N. Russell, Data tables for Lorentz and CTP violation, arXiv:0801.0287 [hep-ph].

[2] D. Anselmi and M. Halat, Renormalization of Lorentz violating theories, Phys. Rev. D 76 (2007) 125011 and arXiv:0707.2480 [hep-th].

[3] D. Anselmi, Weighted power counting and Lorentz violating gauge theories. I: General properties, Ann. Phys. 324 (2009) 874 and arXiv:0808.3470 [hep-th].

[4] D. Anselmi, Weighted power counting and Lorentz violating gauge theories. II: Classification, Ann. Phys. 324 (2009) 1058 and arXiv:0808.3474 [hep-th].

[5] D. Anselmi, Weighted power counting, neutrino masses and Lorentz violating extensions of the Standard Model, Phys. Rev. D 79 (2009) 025017 and arXiv:0808.3475 [hep-ph].

[6] D. Anselmi, Standard Model Without Elementary Scalars And High Energy Lorentz Violation, Eur. Phys. J. C 65 (2010) 523 and arXiv:0904.1849 [hep-ph].

[7] O. Gagnon and G. Moore, Limits on Lorentz violation from highest energy cosmic rays, Phys. Rev. D 70 (2004) 065002 and arXiv:hep-ph/0404196

[8] D. Anselmi and M. Taiuti, Renormalization of high-energy Lorentz violating QED, Phys. Rev. D 81 (2010) 085042 and arXiv:0912.0113 [hep-ph].

[9] B.D. Altschul, Finite duration and energy effects in Lorentz-violating vacuum Cerenkov radiation, Nucl. Phys. B796 (2008) 262 and arXiv:0709.4478 [hep-th]. 
[10] S.R. Coleman and G.L. Glashow, High-energy tests of Lorentz invariance, Phys. Rev. D59 (1999) 116008 and arXiv:hep-ph/9812418.

[11] R. Lehnert and R. Potting, Vacuum Cerenkov radiation, Phys. Rev. Lett. 93 (2004) 110402 and arXiv:hep-ph/0406128;

R. Lehnert and R. Potting, The Cerenkov effect in Lorentz-violating vacua, Phys. Rev. D70 (2004) 125010, Erratum-ibid. D70 (2004) 129906 and arXiv:hep-ph/0408285;

B.D. Altschul, Vacuum Cerenkov radiation in Lorentz-violating theories Without CPT Violation, Phys. Rev. Lett. 98 (2007) 041603 and arXiv:hep-th/0609030;

B.D. Altschul, Cerenkov radiation in a Lorentz-Violating and birefringent vacuum, Phys. Rev. D75 (2007) 105003 and arXiv:hep-th/0701270.

[12] F.R. Klinkhamer and M. Schreck, New two-sided bound on the isotropic Lorentz-violating parameter of modified Maxwell theory, Phys. Rev. D 78 (2008) 085026 and arXiv:0809.3217 [hep-ph].

[13] J.D. Jackson, Classical electrodynamics, §14.9, John Wiley \& Sons Inc., New York, 1962.

[14] D.J. Bird et al., Detection of a cosmic ray with measured energy well beyond the expected spectral cutoff due to cosmic microwave radiation, Astrophys. J. 441 (1995) 144.

[15] D.R.T. Jones, Two-loop $\beta$ function for a $G_{1} \times G_{2}$ gauge group, Phys. Rev. D 25 (1982) 581 .

[16] F. Jegerlehner, The running fine structure constant $\alpha(E)$ via the Adler function, Nuc. Phys. B - Proc. Supp. 181 (2008) 135 and arXiv:0807.4206 [hep-ph].

[17] K. Nakamura et al. (Particle Data Group), J. Phys. G 37 (2010) 075021.

[18] D.Anselmi and E. Ciuffoli, Low-energy phenomenology of scalarless Standard-Model extensions with high-energy Lorentz violation, Phys. Rev. D 83 (2011) 056005 and arXiv:1101.2014 [hep-ph].

[19] See for example, A. Strumia and F. Vissani, Neutrino masses and mixings and..., arXiv:hep-ph/0606054 [hep-ph]. 